\title{
Efficacy and Safety of Endoscopic Treatment for Gastrointestinal Stromal Tumors in the Upper Gastrointestinal Tract
}

\author{
Cicilia Marcella', Shakeel Sarwar ${ }^{2}$, Hui Ye' and Rui Hua Shi ${ }^{1}$ \\ Department of ${ }^{1}$ Gastroenterology, ${ }^{2}$ Orthopedics, Southeast University Affiliated Zhongda Hospital, Nanjing, China
}

Background/Aims: Endoscopic treatment (ET) has been applied for decades to treat subepithelial tumors, including gastrointestinal stromal tumors (GISTs). However, the efficacy of ET remains debatable. In this study, we evaluated the efficacy and safety of ET for GISTs in the upper gastrointestinal tract.

Methods: This retrospective single-center study included 97 patients who underwent ET. All patients were enrolled from July 2014 to July 2018. Parameters such as demographics, size, resection margin, complications, pathological features, procedure time, total cost, and follow-up were investigated and analyzed.

Results: Our study achieved $100 \%$ en bloc resection and $77.4 \%(72 / 93)$ R0 resection. The most common location was the fundus with a mean tumor size of $2.1 \pm 1.4 \mathrm{~cm}$. The mean age, procedure time, hospital stay, and cost were $59.7 \pm 11.3$ years, $64.7 \pm 35.2$ minutes, 6.8 days, and 5,337 dollars, respectively. According to National Institutes of Health classification, 63 (64.9\%), 26 (26.8\%), 5 (5.2\%), and $3(3.1 \%)$ patients belonged to the very low, low, intermediate, and high risk classification, respectively. Immunohistochemistry results showed a $100 \%$ positive rate of CD34, DOG-1, CD117, and Ki67. A mean follow-up of $21.3 \pm 13.0$ months showed no recurrence or metastasis.

Conclusions: ET is effective and safe for curative removal of GISTs in the upper gastrointestinal tract, and it can be a treatment of choice for patients with no metastasis. Clin Endosc 2020;53:458-465

Key Words: Endoscopic full-thickness resection; Endoscopic submucosal dissection; Endoscopic treatment; Gastrointestinal stromal tumor; Submucosal tunneling endoscopic resection

\section{INTRODUCTION}

Gastrointestinal stromal tumors (GISTs) are the most common subepithelial tumors (SETs) arising from the intestinal cells of Cajal. ${ }^{1-3}$ GISTs have some malignant potential determined by the mitotic index, tumor size, and location. ${ }^{4}$ The principle of GIST treatment is a complete R0 resection of the lesion and avoiding tumor rupture. ${ }^{5}$ Although surgery (open,

Received: June 22, 2019 Revised: November 21, 2019

Accepted: December 23, 2019

Correspondence: Rui Hua Shi

Department of Gastroenterology, Southeast University Affiliated Zhongda Hospital, Ding Jia Qiao no.87, Gulou district, Nanjing 210009, China

Tel: +86-139-5179-9326, Fax: +86-025-8326-2835, E-mail: ruihuashi@126.com ORCID: https://orcid.org/0000-0003-4977-8801

(cc) This is an Open Access article distributed under the terms of the Creative Commons Attribution Non-Commercial License (http://creativecommons.org/ licenses/by-nc/3.0) which permits unrestricted non-commercial use, distribution, and reproduction in any medium, provided the original work is properly cited. laparoscopic, or thoracoscopic) has been the preferred treatment for GISTs, many endoscopic resection techniques have proven that endoscopic treatment (ET) is feasible and safe in treating GISTs in the upper gastrointestinal tract. ${ }^{6-8}$ It has several advantages over surgery, such as short hospital stay, low cost, shorter post-resection time to first liquid diet, shorter operative time, and lower intraoperative bleeding.

Many advanced ET techniques have been developed and refined over the past decades. These include endoscopic band ligation, endoscopic mucosal resection, endoscopic submucosal dissection (ESD), endoscopic full-thickness resection (EFTR), submucosal tunneling endoscopic resection (STER), nonexposed endoscopic wall-inversion surgery, laparoscopic endoscopic cooperative surgery (LECS), and a combination of laparoscopic and endoscopic approaches to neoplasia with a nonexposed technique. ${ }^{9-11}$

However, ET remains a matter of debate. Therefore, in this 
study, we retrospectively analyzed GIST patients who underwent ET (ESD, EFTR, and STER), aiming to demonstrate that ET is technically feasible and safe for resection of GISTs. With the further acquisition of experience in ET, we have applied these techniques to large size GISTs.

\section{MATERIALS AND METHODS}

\section{Inclusion and exclusion criteria}

The inclusion criteria were: (1) confirmed diagnoses of GIST by histopathologic evaluation; (2) GIST patients with no age, gender, or tumor size limitations; (3) eligibility for ET; (4) location in the upper gastrointestinal tract; and (5) complete data available. The exclusion criteria were: (1) evidence of lymph node involvement or distant metastasis; (2) coexistence of serious infection, multiple organ failure, or other malignant tumors; (3) patients who underwent laparoscopic or open surgery; and (4) incomplete data.

\section{Patients}

A single-center, retrospective study was carried out in the Southeast University-affiliated with Zhongda Hospital (Nanjing, China) between July 2014 to July 2018. A total of 143 patients were confirmed histopathologically as having GIST. After exclusion, 97 patients were selected (Fig. 1). Preoperatively, all patients were evaluated by medical history, electrocardiography, laboratory tests, computed tomography (CT), gastroscopy, and endoscopic ultrasound (EUS) to determine the exact size, layer of origin, blood vessel involvement, margin, and growth pattern.

The institutional review board and ethical committee of the
Southeast University-affiliated Zhongda Hospital approved the study protocol. All patients provided written informed consent and were told of the risks and benefits of the procedure.

\section{Endoscopic treatment procedure}

All procedures were done under general anesthesia with intubation by a skilled endoscopist. During the procedures, vital signs including heart rate, blood pressure, and $\mathrm{Sp}_{2}$ were monitored continuously.

\section{Endoscopic submucosal dissection and endoscopic full-thickness resection}

ESD and EFTR standard procedures were as follow: the transparent cap was attached for a better view; the lesion boundaries were marked by argon plasma coagulation (APC); a mixture solution of saline $(100 \mathrm{~mL})$, indigo carmine dye $(2 \mathrm{~mL})$, and epinephrine $(1 \mathrm{~mL})$ was injected into the submucosa of the marked lesion; the initial incision of the mucosal and submucosal layer around the lesion was performed with a hook knife (KD-620LR; Olympus, Aomori, Japan); the tumor was dissected according to the tumor origin to ensure complete en bloc resection using an IT knife 2 (KD- 611L; Olympus) or IT knife (KD-610L; Olympus); and final closure of the surgical wound using metal clips was applied. The only difference was that the EFTR procedure was used for deeper resection of the tumor including an incision of the serosal layer to generate artificial perforation. Perforated gastric wall defects (GWDs) were closed with endoscopic clips. If the GWD was too large, a nylon band was placed over the GWD and fixed with the help of clips around the normal mucous membrane by dual-channel gastroscopy (GIF-2TQ260M; Olympus; Fig. 2). The GWD was entirely closed by tightening the nylon

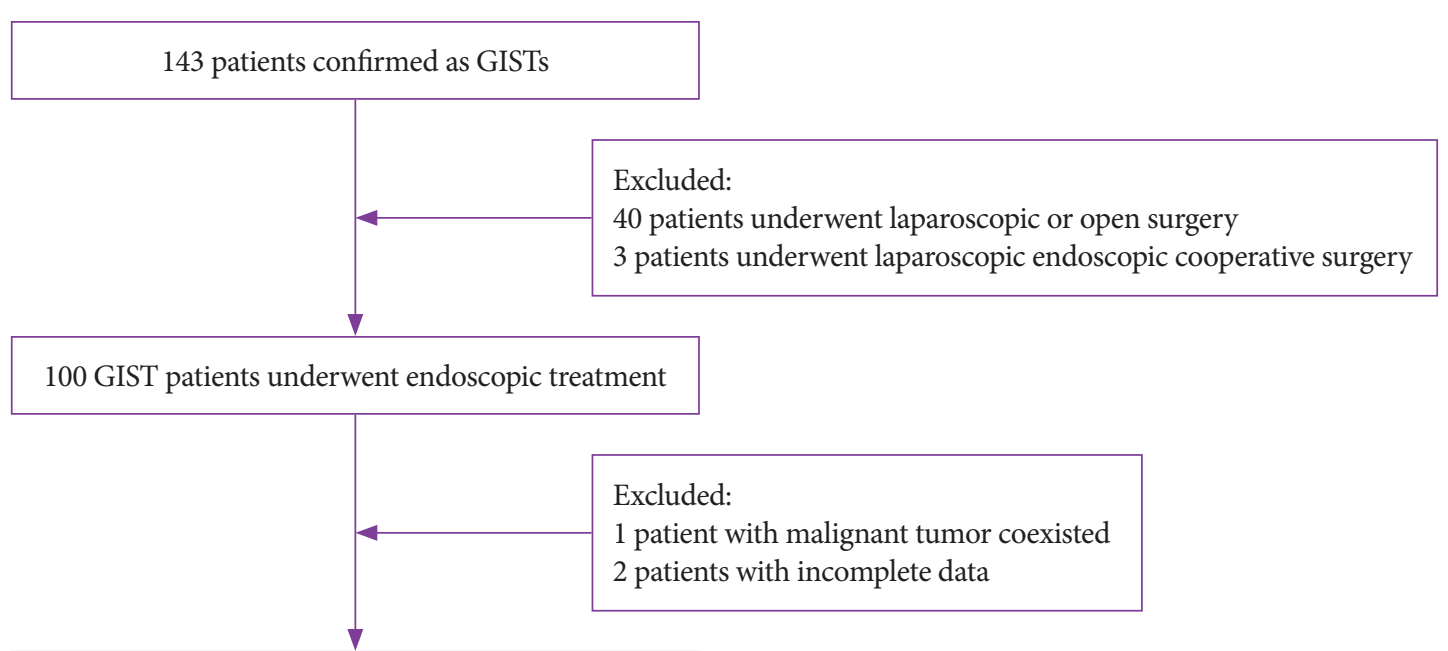


band and releasing the grasper. For all patients who developed intraoperative pneumoperitoneum, a 20/21-gauge needle was inserted percutaneously to release the peritoneum tension. If bleeding or perforation failed to be managed endoscopically, an emergency conversion of laparoscopic or open surgery was indicated.

\section{Submucosal tunneling endoscopic resection}

STER standard procedures included: injecting a mixture solution of saline $(100 \mathrm{~mL})$, indigo carmine dye $(2 \mathrm{~mL})$, and epinephrine $(1 \mathrm{~mL})$ into the submucosal layer; creating an initial incision $5 \mathrm{~cm}$ proximal to the tumor followed by a tunnel between the mucosal and muscularis propria layers with a hook knife (KD-620LR; Olympus); tumor dissection with a snare or IT knife 2 (KD-611L; Olympus) from the muscular layer, and closure of the mucosal entry orifice with hemostatic clips.

\section{Post-operative treatment}

After the procedure, antibiotics, proton pump inhibitors, and hemostatic drugs were routinely administered to prevent infection and delayed bleeding. All patients were told to maintain strict bed rest for $24 \mathrm{~h}$ and fast for at least $24 \mathrm{~h}$ (ESD, STER) and $48 \mathrm{~h}$ (EFTR). Postoperative time to first liquid diet depended on the patient's complete blood count and whether fever occurred or not. Body temperature, abdominal pain or distention, presence of flatulence or stool passing, and signs of peritonitis or hemorrhage were monitored. A gastric decompression tube was used for patients in whom perforation occurred. After discharge, patients were prescribed a proton pump inhibitor taken orally for eight weeks and additional liquid Chinese medicine to improve mucosal healing. According to the type of KIT/PDGFRA mutation, patients with intermediate or high risk were recommended adjuvant imatinib therapy (6-12 months) even though R0 resection was achieved.

\section{Histopathology}

Tissue specimens were analyzed with immunohistochemistry staining of CD34, CD117, DOG-1, Ki67, smooth muscle actin, desmin, and S-100. Immunohistochemistry-positive staining for CD34, CD117, and DOG-1 was considered to be GIST. Tumor size, resection margin, and mitotic index were evaluated. Complete resection was achieved upon en bloc tumor removal and tumor-free margins in the pathologic examination. The risk assessment of GISTs was determined according to the National Institutes of Health (NIH) consensus and classification. Assessment was based on the tumor size and mitotic index and categorized as very low risk, low risk, intermediate risk, and high risk.
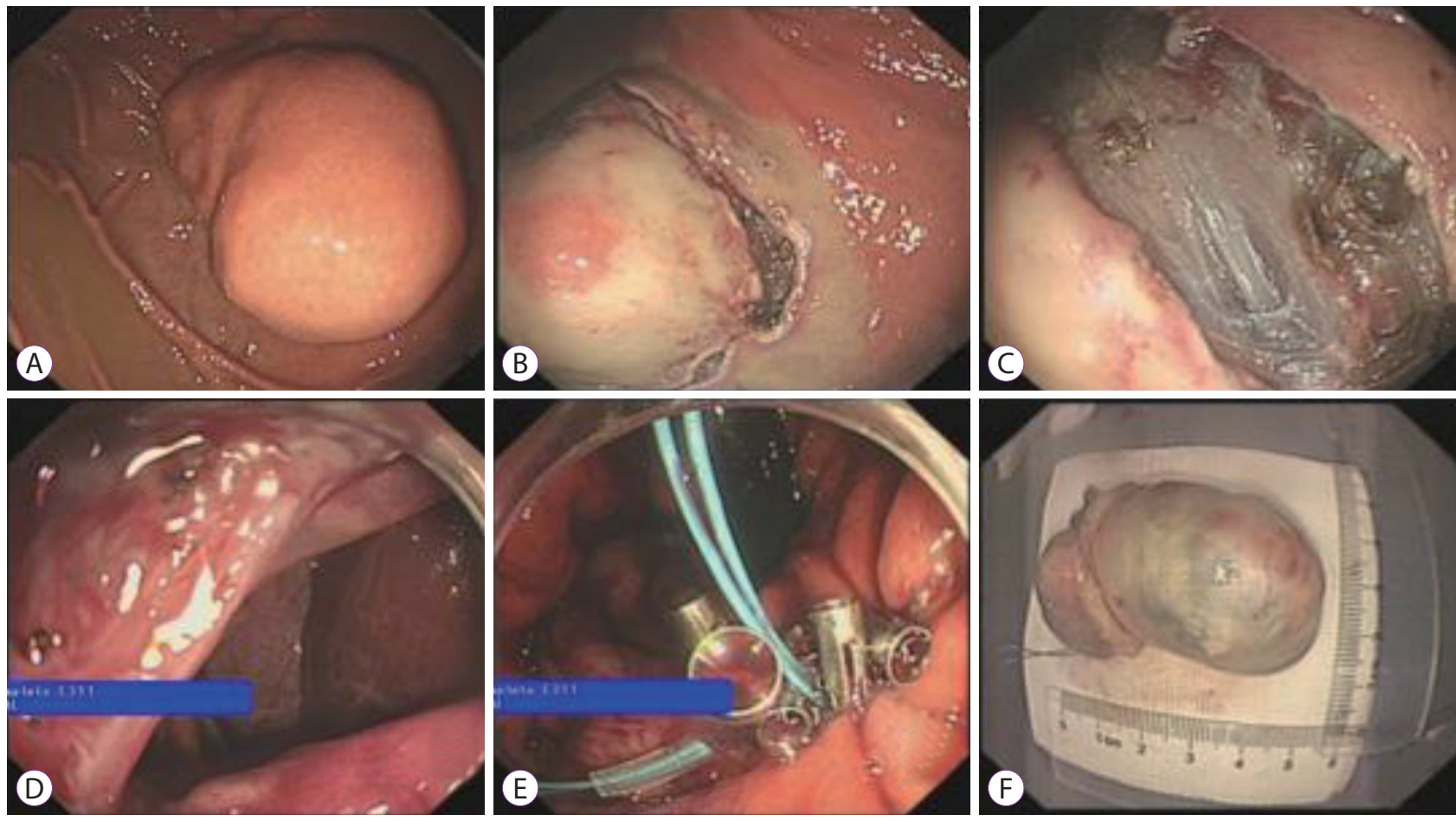

Fig. 2. Endoscopic full-thickness resection treatment of the gastric stromal tumor. (A) A large gastrointestinal stromal tumor located at the fundus of the stomach by gastroscope. (B) Mucosal incision with a hook knife. (C) The lesion is dissected from the deeper fibers of the muscularis propria. (D) The tumor is completely resected, creating an artificial perforation. (E) The wound is closed with a nylon band and several clips. (F) The resected specimen. 


\section{Follow-up}

All patients were observed with standard upper gastrointestinal endoscopy at 3, 6, and 12 months after ET, and then annually for three years. For high risk patients, contrast-enhanced CT scan will be obtained to exclude any metastasis every 3-6 months after the procedure for three years, followed by biannual examination for five years. For low risk patients, CT scans can be obtained every 6 to 12 months for five years.

Table 1. Detailed Characteristic of the 97 Enrolled Patients

\begin{tabular}{|c|c|c|}
\hline Parameters & $n$ & $\%$ \\
\hline \multicolumn{3}{|l|}{ Age } \\
\hline Mean (range) & $59.7 \pm 11.3$ & $23-87$ \\
\hline \multicolumn{3}{|l|}{ Tumor size } \\
\hline$\leq 2 \mathrm{~cm}$ & 65 & 67.0 \\
\hline$>2-\leq 5 \mathrm{~cm}$ & 29 & 29.9 \\
\hline$>5 \mathrm{~cm}$ & 3 & 3.1 \\
\hline \multicolumn{3}{|l|}{ Gender } \\
\hline Male & 51 & 53.6 \\
\hline Female & 46 & 46.4 \\
\hline \multicolumn{3}{|l|}{ Tumor location } \\
\hline Esophagus & 2 & 2.1 \\
\hline Cardia & 6 & 6.2 \\
\hline Fundus & 64 & 66.0 \\
\hline Body & 18 & 18.6 \\
\hline Antrum & 7 & 7.2 \\
\hline \multicolumn{3}{|l|}{ Layer of origin } \\
\hline Submucosa & 7 & 7.2 \\
\hline Muscularis propria & 90 & 92.8 \\
\hline \multicolumn{3}{|l|}{ Symptoms } \\
\hline Asymptomatic & 35 & 36.1 \\
\hline Dysphagia & 1 & 1.0 \\
\hline Abdominal pain & 38 & 39.2 \\
\hline Abdominal distention & 10 & 10.3 \\
\hline Melena & 4 & 4.1 \\
\hline Others & 9 & 9.3 \\
\hline \multicolumn{3}{|l|}{ Excavation procedure } \\
\hline ESD & 48 & 49.5 \\
\hline EFTR & 46 & 47.4 \\
\hline STER & 3 & 3.1 \\
\hline \multicolumn{3}{|l|}{ Risk classification } \\
\hline Very low & 63 & 64.9 \\
\hline Low & 26 & 26.8 \\
\hline Moderate & 5 & 5.2 \\
\hline High & 3 & 3.1 \\
\hline \multicolumn{3}{|l|}{ Mitotic index } \\
\hline$\leq 5 / 50$ high-power field & 91 & 93.8 \\
\hline$>5 / 50$ high-power field & 6 & 6.2 \\
\hline
\end{tabular}

EFTR, endoscopic full-thickness resection; ESD, endoscopic submucosal dissection; STER, submucosal tunneling endoscopic resection.

\section{Statistical analysis}

All statistical analyses were performed using SPSS 21.0 statistics software (SPSS Inc., Chicago, IL, USA). Continuous data are presented as a mean \pm standard deviation and analyzed using a Student's $t$-test and categorical data are displayed as number $(n)$ and percentage (\%) and calculated using Fisher's exact test or a chi-squared test. A two-sided $p<0.05$ was considered statistically significant.

\section{RESULTS}

\section{Clinical characteristics}

This study consisted of 97 patients, 51 of whom were males (53.6\%). The mean age was $59.7 \pm 11.3$ years (range, $23-87$ years). Of these 97 patients, 2 GISTs were located in the esophagus, 6 in the cardia, 64 in the fundus, 19 and 6 in the body and antrum of the stomach, respectively. The mean tumor size was $2.1 \pm 1.4 \mathrm{~cm}$ (range, $0.4-8 \mathrm{~cm}$ ). EUS was used to analyze the layer of origin of the tumor, the submucosa $(n=7,7.2 \%)$, and the muscularis propria $(n=90,92.8 \%)$. The demographic and clinical features of all included patients are summarized in Table 1.

\section{Therapeutic outcomes and histopathologic evalua- tion}

Of the 97 patients, 48, 46, and 3 patients underwent ESD, EFTR, and STER procedures, respectively. The mean operative time was $64.7 \pm 35.2$ minutes (range, $25-180$ minutes), and the mean length of stay was $6.8 \pm 2.4$ days (range, 3-16 days). The mean cost for ET was 5,337 $\pm 1,568$ dollars. The prevalence of en bloc resection was $100 \%$ (97/97), and the complete resection rate was $77.4 \%$ (72/93; Table 2). Four patients did not undergo tumor-free margin assessment due to broken specimens.

Table 2. Outcomes of Endoscopic Treatment for Gastrointestinal Stromal Tumor in the Upper Gastrointestinal Tract

\begin{tabular}{lc}
\hline Parameters & $\boldsymbol{n}$ \\
\hline En bloc resection & $97(100 \%)$ \\
Complete resection & $72(77.4 \%)^{\mathrm{a})}$ \\
\hline Mean operative time (min) & $64.7 \pm 35.2$ \\
Mean length of stay (days) & $6.8 \pm 2.4$ \\
Mean cost (\$) & $5,337 \pm 1,568$ \\
Complications & 8 \\
Intraoperative massive bleeding & 1 \\
Intraoperative perforation & 7 \\
Mean of follow-up (mo) & $21.3 \pm 13.0$ \\
\hline
\end{tabular}

${ }^{\text {a) }}$ Four patients were failed to assessed the tumor-free margin due to broken specimens. 
Larger resected tumors were cut into two or more pieces by snare due to the difficulty in passing the resected tumor peroral. The complete resection rate was higher in females (39/46, $84.8 \%$ ) than in males $(33 / 51,64.7 \%$; $p=0.049)$. Further largescale studies of the complete resection rate between males and females are required. No significant correlation was observed between the complete resection rate and other clinical characteristics, such as age, tumor size, the layer of origin, and mitotic index (Table 3).

When we compared the results between the three procedures, the complete resection (R0) rate of STER, EFTR, and ESD was $100 \%(2 / 2), 84.1 \%$ (37/44) and 70.8\% (34/48), respectively. Note that four out of ninety-seven resected tumors were not assessed due to damaged specimens. STER and EFTR showed higher rates of complete resection than the ESD group. However, STER had a small sample size; thus, further investigation is needed to confirm the outcome. The mean operation time ( $\mathrm{min}$ ) of STER, EFTR, and ESD was $38 \pm 10$ (range, 30-50), 68 \pm 37 (range, 25-165), and 62 \pm 32 (range, 25-180), respectively. Whereas the mean length of hospitalization of the three procedures was as follows: $5.7 \pm 2.5$ days (STER), $6.7 \pm 2.4$ days (EFTR), and $6.8 \pm 2$ days (ESD).

The resected specimens showed $100 \%$ positive rate of CD34, DOG-1, CD117, and Ki67. According to the NIH classification, 63 (64.9\%), 26 (26.8\%), 5 (5.2\%), and 3 (3.1\%) patients belonged to the very low, low, intermediate, and high risk classifications, respectively. The mitotic index in 6 patients was $>5$ mitoses per 50 high-power field (Table 3 ).

\section{Complications}

Eight patients in the ESD group experienced complications, including seven intraoperative perforations and one massive bleeding. No apparent adverse events were observed during EFTR or STER procedures. All patients with a perforation underwent successful repair using clips and a nylon band, except one patient who was converted to emergency laparoscopic surgery due to an uncontrolled perforation. During hospitalization, delayed pneumoperitoneum occurred in one patient $(1.0 \%)$ and was treated by laparoscopic repair. One patient developed postoperative peritonitis that was controlled by conservative medical therapy. Minor bleeding occurred in most cases, but hemostasis was achieved by hot biopsy forceps or APC. No patient had other complications such as delayed bleeding, deep venous thrombosis, myocardial infarction, or subcutaneous emphysema.

\section{Follow-up outcomes}

During the mean follow-up of $21.3 \pm 13.0$ months (range, 3-48 months), no local recurrence or distant metastasis was observed in any patient. Three patients were lost to follow-up because the patients are staying away from the hospital and we weren't able to access them through phone calls.

\section{DISCUSSION}

GISTs are the most common mesenchymal tumor of the gastrointestinal tract. ${ }^{12}$ They are thought to arise from the

Table 3. Comparisons of Rates of Complete Resection and Complications of Endoscopic Treatment

\begin{tabular}{|c|c|c|c|c|c|c|}
\hline \multirow{2}{*}{ Factors } & \multicolumn{3}{|c|}{ Complete resection $(n=93)^{a)}$} & \multicolumn{3}{|c|}{ Complications $(n=97)$} \\
\hline & Yes & No & $p$-value & Yes & No & $p$-value \\
\hline Age (yr) & & & 0.465 & & & 0.486 \\
\hline$<60$ & 34 & 12 & & 5 & 43 & \\
\hline$\geq 60$ & 38 & 9 & & 3 & 46 & \\
\hline Gender & & & 0.049 & & & 0.471 \\
\hline Male & 33 & 15 & & 3 & 48 & \\
\hline Female & 39 & 6 & & 5 & 41 & \\
\hline Tumor size $(\mathrm{cm})$ & & & 0.108 & & & 1 \\
\hline$\leq 2$ & 48 & 18 & & 5 & 60 & \\
\hline$>2$ & 24 & 3 & & 3 & 29 & \\
\hline Layer of origin & & & 0.188 & & & 0.464 \\
\hline Submucosa & 4 & 3 & & 1 & 6 & \\
\hline Muscularis propria & 68 & 18 & & 7 & 83 & \\
\hline Mitotic index & & & 1 & & & 1 \\
\hline$\leq 5 / 50$ high-power field & 68 & 20 & & 8 & 83 & \\
\hline$>5 / 50$ high-power field & 4 & 1 & & 0 & 6 & \\
\hline
\end{tabular}

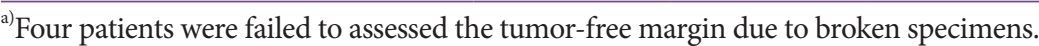


intestinal cells of Cajal or primitive pluripotent stem cells and mostly occur in middle-aged or older people with no gender predilection. ${ }^{13}$ All GISTs can have some malignant potential, even small tumors with low mitotic rates have been observed to be malignant. ${ }^{8}$ According to National Comprehensive Cancer Network guidelines, all GISTs $>2 \mathrm{~cm}$ in size should be resected. However, many GISTs $<2 \mathrm{~cm}$ are found incidentally with endoscopy and treatment options include resection and periodic surveillance by endoscopy. Repeated endoscopic procedures may induce patient anxiety, reduce cost-effectiveness, increase the risks associated with an endoscopic procedure, and a delayed diagnosis of malignant tumors. ${ }^{14}$ Thus, some guidelines suggest resection of tumors $<2 \mathrm{~cm}$ in size. . $^{15,16}$

For decades, surgical resection (open or laparoscopic) has been the treatment of preference for GISTs. Laparoscopic surgery has been considered to have some benefits over open surgery such as low morbidity, short hospital stays, and longterm disease-free survival. ${ }^{17}$ Hiki et al. ${ }^{18}$ developed the LECS technique in 2008. This procedure is a combination of laparoscopic surgery with the assistance of a gastroscope in order to preserve the gastric wall. Namikawa and Hanazaki ${ }^{19}$ concluded that the LECS method has the advantage of a reduced resected area and lower estimated blood loss when compared to laparoscopic surgery. Sometimes laparoscopic procedure alone has some limitations like an inability to localize the resection area, and when the tumor is intracavitary, identifying tumors from the serosal side may be challenging. Moreover, a tumor located in the esophagus or near the esophagus-gastric junction may be challenging to perform with laparoscopic surgery. ${ }^{20}$

Despite the rapid development of endoscopic technology in recent years, surgery is still preferred for the removal of SETs $>2 \mathrm{~cm}$, while ET is favored for removing SETs $<2 \mathrm{~cm}$. ${ }^{21}$ Many studies have reported the efficacy and safety of ET in treating gastric SETs. When compared to ET, surgical resection causes more extensive wounds, higher costs, a longer recovery time, and lower quality of life..$^{22-27}$ Yin et al. ${ }^{6}$ evaluated three different methods (ESD, LECS, and laparoscopic resection) for GIST $\leq 5 \mathrm{~cm}$. They found that the operative time and intraoperative blood loss in the ESD group were significantly less than in the LECS and laparoscopic resection groups. Therefore, the endoscopic approach has some benefits over laparoscopic or open surgery to some extent.

STER was initially reported by Xu et al. ${ }^{28}$ in 2012 for the resection of SETs, which was inspired by the digestive endoscopic tunnel technique. Some retrospective studies have demonstrated the efficacy and feasibility of STER for esophageal and gastric SETS. ${ }^{29-31} \mathrm{Li}$ et al. ${ }^{30}$ evaluated 32 cases of gastric SETs (11 cases were GIST) managed by STER. It achieved $100 \%$ en bloc resection and no recurrence was observed.
Tan et al. ${ }^{31}$ reported 20 cases of gastric GISTs, 95\% of which achieved successful en bloc resection with a comparatively low complication rate without any surgery conversion. STER was regarded to be superior to ESD or surgical methods in treating upper gastrointestinal SETs based on its benefits in maintaining mucosal integrity, faster healing, and reduced risks of perforation, infection, and esophagus stricture. ${ }^{32,33}$ In the present study, we used the STER technique to treat 3 cases located in the esophagus and cardia. No postoperative complications or recurrence was detected.

The common complications related to ET were massive bleeding and perforation. In our study, the perforation rate was $7.2 \%$ (7/97); a majority of cases $(n=6,85.7 \%)$ were treated endoscopically. In one patient, the tumor located in the fundus was large and tightly adhered to the third layer. Perforation occurrence was large; thus, conversion to emergency laparoscopic surgery was required. Perforation or bleeding is sometimes an inevitable complication even in the hands of an expert endoscopist. Some measures should be taken during the procedure to prevent massive bleeding such as pre-operative surveillance of the blood vessel flow by contrast-enhanced $\mathrm{EUS}^{34}$; during the procedure, minute or larger vessels should be directly coagulated to prevent blind endoscopic manipulation; flushing of icy saline solution containing norepinephrine and APC or hot biopsy forceps can be used to stop the bleeding and metal clips can be applied to occlude vessels. During ET, such as resection that does not involve full mucosal thickness resection, avoidance of perforation must be solicited. If perforation occurs, prevention of peritoneal seeding is necessary. Some methods including proper grasping of the resected tumor and complete closure of perforations with emerging closing techniques like the Overstitch endoscopic suturing system, ${ }^{35}$ over-the-scope clips, ${ }^{36}$ string clip suturing, ${ }^{37}$ slipknot clip suturing, ${ }^{38-40}$ and the hold-and-drag closure technique ${ }^{41}$ can be applied.

In this study, we sought to evaluate ET safety and feasibility in treating upper GISTs. All patients underwent a single, successful $100 \%$ en bloc resection of the lesion, including 28 cases with tumor diameters between $2-5 \mathrm{~cm}$ and 3 cases $>5 \mathrm{~cm}$. Overall, 77.4\% (72/93) complete resection was achieved, and an overall complication rate of $8.2 \%(8 / 97)$, which was lower than the rates reported for surgical resection. After a follow up for $21.3 \pm 13.0$ months (range, 3-48 months), there was no recurrence or metastasis. These results indicated that ET application is feasible and can be regarded as the treatment of choice for GISTs in the upper gastrointestinal tract. Although resection of a larger upper GIST by ET remains controversial, to our experience, ET is feasible for treating GISTs up to $8 \mathrm{~cm}$ (the largest tumor in our study). Li et al. ${ }^{9}$ demonstrated that there was no significant difference in complications and en 
bloc resection for tumors $<5$ and $\geq 5 \mathrm{~cm}$. This result was in line with our study for tumors $\leq 2$ and $>2 \mathrm{~cm}$ when comparing the complete resection and complication rates. However, efforts must be made by endoscopists to benefit patients by providing an optimal ET choice with minimal complication risks.

In conclusion, our study provides evidence that ET for GISTs in upper gastrointestinal tract is safe and feasible. ET for GIST also achieved relatively high en bloc resection (100\%) and $\mathrm{R} 0$ resection $(77.4 \%)$, with relatively low complication and recurrence rates. ET preserves the normal structure of the esophagus and stomach to maintain the function of the upper gastrointestinal tract and thus improves the patients' quality of life. Limitations to our present study are its retrospective, single-center nature that minimizes its generalizability to all patients who have undergone ET for GISTs. Larger prospective studies are required to support our primary results. Further, a longer duration of follow-up is needed to determine the longterm disease-free survival.

\section{Conflicts of Interest}

The authors have no financial conflicts of interest.

\section{ORCID}

Cicilia Marcella: https://orcid.org/0000-0003-0511-144X

Shakeel Sarwar: https://orcid.org/0000-0001-8482-2711

Hui Ye: https://orcid.org/0000-0003-2729-9244

\section{REFERENCES}

1. Nilsson B, Bümming P, Meis-Kindblom JM, et al. Gastrointestinal stromal tumors: the incidence, prevalence, clinical course, and prognostication in the preimatinib mesylate era--a population-based study in western Sweden. Cancer 2005;103:821-829.

2. Alvarado-Cabrero I, Vázquez G, Sierra Santiesteban FI, Hernández-Hernández DM, Pompa AZ. Clinicopathologic study of 275 cases of gastrointestinal stromal tumors: the experience at 3 large medical centers in Mexico. Ann Diagn Pathol 2007;11:39-45.

3. Chhoda A, Jain D, Surabhi VR, Singhal S. Contrast enhanced harmonic endoscopic ultrasound: a novel approach for diagnosis and management of gastrointestinal stromal tumors. Clin Endosc 2018;51:215-221.

4. Fletcher CD, Berman JJ, Corless C, et al. Diagnosis of gastrointestinal stromal tumors: a consensus approach. Int J Surg Pathol 2002;10:81-89.

5. ESMO/European Sarcoma Network Working Group. Gastrointestinal stromal tumours: ESMO clinical practice guidelines for diagnosis, treatment and follow-up. Ann Oncol 2014;25 Suppl 3:iii21-iii26.

6. Yin X, Yin Y, Chen H, et al. Comparison analysis of three different types of minimally invasive procedures for gastrointestinal stromal tumors $\leq 5$ cm. J Laparoendosc Adv Surg Tech A 2018;28:58-64.

7. He G, Wang J, Chen B, et al. Feasibility of endoscopic submucosal dissection for upper gastrointestinal submucosal tumors treatment and value of endoscopic ultrasonography in pre-operation assess and post-operation follow-up: a prospective study of 224 cases in a single medical center. Surg Endosc 2016;30:4206-4213.

8. Andalib I, Yeoun D, Reddy R, Xie S, Iqbal S. Endoscopic resection of gastric gastrointestinal stromal tumors originating from the muscularis propria layer in North America: methods and feasibility data. Surg En- dosc 2018;32:1787-1792.

9. Li J, Tang J, Lua GW, et al. Safety and efficacy of endoscopic submucosal dissection of large $(\geq 3 \mathrm{~cm})$ subepithelial tumors located in the cardia. Surg Endosc 2017;31:5183-5191.

10. Kim HH. Endoscopic treatment for gastrointestinal stromal tumor: advantages and hurdles. World J Gastrointest Endosc 2015;7:192-205.

11. Marcella C, Shi RH, Sarwar S. Clinical overview of GIST and its latest management by endoscopic resection in upper GI: a literature review. Gastroenterol Res Pract 2018;2018:6864256.

12. Miettinen M, Sobin LH, Lasota J. Gastrointestinal stromal tumors of the stomach: a clinicopathologic, immunohistochemical, and molecular genetic study of 1765 cases with long-term follow-up. Am J Surg Pathol 2005;29:52-68.

13. DeMatteo RP, Lewis JJ, Leung D, Mudan SS, Woodruff JM, Brennan MF. Two hundred gastrointestinal stromal tumors: recurrence patterns and prognostic factors for survival. Ann Surg 2000;231:51-58.

14. Demetri GD, von Mehren M, Antonescu CR, et al. NCCN task force report: update on the management of patients with gastrointestinal stromal tumors. J Natl Compr Canc Netw 2010;8 Suppl 2:S1-S41; quiz S42-S44.

15. Nishida T, Hirota S, Yanagisawa A, et al. Clinical practice guidelines for gastrointestinal stromal tumor (GIST) in Japan: English version. Int J Clin Oncol 2008;13:416-430.

16. Puchalski CM. Spirituality in the cancer trajectory. Ann Oncol 2012;23 Suppl 3:49-55.

17. Miettinen M, Lasota J. Gastrointestinal stromal tumors: pathology and prognosis at different sites. Semin Diagn Pathol 2006;23:70-83.

18. Hiki N, Yamamoto Y, Fukunaga T, et al. Laparoscopic and endoscopic cooperative surgery for gastrointestinal stromal tumor dissection. Surg Endosc 2008;22:1729-1735.

19. Namikawa T, Hanazaki K. Laparoscopic endoscopic cooperative surgery as a minimally invasive treatment for gastric submucosal tumor. World J Gastrointest Endosc 2015;7:1150-1156.

20. He Z, Sun C, Zheng Z, et al. Endoscopic submucosal dissection of large gastrointestinal stromal tumors in the esophagus and stomach. J Gastroenterol Hepatol 2013;28:262-267.

21. Zhang J, Huang K, Ding S, et al. Clinical applicability of various treatment approaches for upper gastrointestinal submucosal tumors. Gastroenterol Res Pract 2016;2016:9430652.

22. Chun SY, Kim KO, Park DS, et al. Endoscopic submucosal dissection as a treatment for gastric subepithelial tumors that originate from the muscularis propria layer: a preliminary analysis of appropriate indications. Surg Endosc 2013;27:3271-3279.

23. Lee CM, Kim HH. Minimally invasive surgery for submucosal (subepithelial) tumors of the stomach. World J Gastroenterol 2014;20:1303513043.

24. Chu YY, Lien JM, Tsai MH, et al. Modified endoscopic submucosal dissection with enucleation for treatment of gastric subepithelial tumors originating from the muscularis propria layer. BMC Gastroenterol 2012;12:124.

25. Zhang Y, Ye LP, Zhou XB, et al. Safety and efficacy of endoscopic excavation for gastric subepithelial tumors originating from the muscularis propria layer: results from a large study in China. J Clin Gastroenterol 2013;47:689-694.

26. Inoue $\mathrm{H}$, Ikeda $\mathrm{H}$, Hosoya $\mathrm{T}$, et al. Submucosal endoscopic tumor resection for subepithelial tumors in the esophagus and cardia. Endoscopy 2012;44:225-230.

27. Schmidt A, Bauder M, Riecken B, von Renteln D, Muehleisen H, Caca K. Endoscopic full-thickness resection of gastric subepithelial tumors: a single-center series. Endoscopy 2015;47:154-158.

28. Xu MD, Cai MY, Zhou PH, et al. Submucosal tunneling endoscopic resection: a new technique for treating upper GI submucosal tumors originating from the muscularis propria layer (with videos). Gastrointest Endosc 2012;75:195-199.

29. Lu J, Zheng M, Jiao T, Wang Y, Lu X. Transcardiac tunneling technique 
for endoscopic submucosal dissection of gastric fundus tumors arising from the muscularis propria. Endoscopy 2014;46:888-892.

30. Li QL, Chen WF, Zhang C, et al. Clinical impact of submucosal tunneling endoscopic resection for the treatment of gastric submucosal tumors originating from the muscularis propria layer (with video). Surg Endosc 2015;29:3640-3646

31. Tan Y, Tang X, Guo T, et al. Comparison between submucosal tunneling endoscopic resection and endoscopic full-thickness resection for gastric stromal tumors originating from the muscularis propria layer. Surg Endosc 2017;31:3376-3382.

32. Gong W, Xiong Y, Zhi F, Liu S, Wang A, Jiang B. Preliminary experience of endoscopic submucosal tunnel dissection for upper gastrointestinal submucosal tumors. Endoscopy 2012;44:231-235.

33. Ye LP, Zhang Y, Mao XL, Zhu LH, Zhou X, Chen JY. Submucosal tunneling endoscopic resection for small upper gastrointestinal subepithelial tumors originating from the muscularis propria layer. Surg Endosc 2014;28:524-530.

34. Tamura T, Kitano M. Contrast enhanced endoscopic ultrasound imaging for gastrointestinal subepithelial tumors. Clin Endosc 2019;52:306313.

35. Kantsevoy SV, Bitner M, Mitrakov AA, Thuluvath PJ. Endoscopic su- turing closure of large mucosal defects after endoscopic submucosal dissection is technically feasible, fast, and eliminates the need for hospitalization (with videos). Gastrointest Endosc 2014;79:503-507.

36. Vance RB, Agrawal D. Endoscopic repair of gastric perforation with an over-the-scope clip after endoscopic mucosal resection. Gastrointest Endosc 2014;80:1205-1206.

37. Yahagi N, Nishizawa T, Akimoto T, Ochiai Y, Goto O. New endoscopic suturing method: string clip suturing method. Gastrointest Endosc 2016;84:1064-1065.

38. Nishizawa T, Uraoka T, Suzuki H, et al. New endoscopic suturing method: slip knot clip suturing. Gastrointest Endosc 2015;82:570-571.

39. Nishizawa T, Uraoka T, Sagara S, et al. Endoscopic slipknot clip suturing method: an ex vivo feasibility study (with video). Gastrointest Endosc 2016;83:447-450.

40. Nishizawa T, Ochiai Y, Uraoka T, et al. Endoscopic slip-knot clip suturing method: prospective pilot study (with video). Gastrointest Endosc 2017;85:433-437.

41. Akimoto T, Goto O, Sasaki M, et al. "Hold-and-drag" closure technique using repositionable clips for large mucosal defects after colonic endoscopic submucosal dissection. Endosc Int Open 2016;4:E1068-E1072. 